\title{
Three-dimensional simulations of blob dynamics in a simple magnetized torus
}

\author{
Federico D. Halpern, ${ }^{\text {a) }}$ Annalisa Cardellini, ${ }^{\text {b) }}$ Paolo Ricci, Sébastien Jolliet, Joaquim Loizu, \\ and Annamaria Mosetto \\ École Polytechnique Fédérale de Lausanne (EPFL), Centre de Recherches en Physique des Plasmas, \\ Association Euratom-Confédération Suisse, CH-1015 Lausanne, Switzerland
}

(Received 16 November 2013; accepted 15 January 2014; published online 10 February 2014)

The propagation of blobs, structures of localized enhanced plasma pressure, is studied in global three-dimensional simulations of a simple magnetized torus. In particular, we carry out single-seeded blob simulations to explore the dependence of the blob velocity with respect to its size. It is found that the velocity scaling for two-dimensional blobs is satisfied in the parameter space where polarization currents are the dominant damping mechanism. On the other hand, three-dimensional blobs propagate faster than their two-dimensional counterparts in the parallel current damping regime. A detailed analysis of the charge and current balance reveals that, in fact, the difference in speed is due to an overestimation of the strength of the sheath current term in the two-dimensional model compared to the self-consistent three-dimensional model. [http://dx.doi.org/10.1063/1.4864324]

\section{INTRODUCTION}

Turbulence at the edge of many laboratory plasmas is characterized by the presence of blobs, structures of enhanced plasma pressure compared to the background, localized in the plane perpendicular to the magnetic field and extended along it. ${ }^{1-3}$ Blobs can move across the magnetic field and travel as individual entities over a distance of several times their size, thus leading to intermittent transport of particles and energy. Their generation and motion have been extensively studied because of their importance in burning plasma experiments and future fusion reactors. An extensive review of blob physics is presented in Refs. 3 and 4.

The propagation of blobs can be understood as follows. Magnetic field curvature and gradients due to the blob spatial inhomogeneity induce charge-dependent drifts leading to charge accumulation. The resulting electric field gives rise to an $\mathbf{E} \times \mathbf{B}$ drift, responsible for the blob motion, whose amplitude depends on the available current path to damp charge accumulation.

The mechanisms for the damping of charge accumulation have been studied by using a two-dimensional model, based on the quasi-neutrality equation, typically assuming homogeneous plasma along the magnetic field lines and sheath current given by Bohm's condition at the field line ends. A detailed discussion of the different damping mechanisms can be found in Ref. 4. The most basic model shows that two damping mechanisms are active, depending on $a_{\perp}$, the blob size along the direction perpendicular to both $\mathbf{B}$ and $\nabla B$, normalized to $a_{0}=\left[4\left(L_{c}^{2} / R^{2}\right)\left(R / \rho_{s}\right)\right]^{1 / 5} \rho_{s}$ (here $L_{c}$ is the magnetic field connection length, $\rho_{s}$ is the ion sound Larmor radius, and $R$ is the curvature and typical variation scale length of the magnetic field.). If $a_{\perp} \lesssim 1$, charge accumulation is damped by cross-field polarization current and the

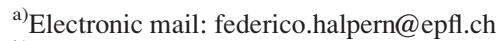

${ }^{b)}$ Also at Department of Energy, Politecnico di Torino, Corso Duca degli Abruzzi 24, 10129 Torino, Italy.
}

blob velocity is proportional to $\sqrt{a_{\perp}}$. On the other hand, if $a_{\perp} \gtrsim 1$, charge accumulation is damped by parallel currents that flow to the vessel, through the plasma sheath, and the blob velocity is proportional to $1 / a_{\perp}^{2}$. The two regimes have been unified into a general scaling law that has been compared with experimental measurements of blob velocity taken on the TORPEX simple magnetized torus (SMT), ${ }^{5,6}$ showing good agreement. ${ }^{7}$ The scaling has also been verified against non-linear two-dimensional simulations carried out in the plane perpendicular to the magnetic field. ${ }^{8}$

As a matter of fact, while most of the blob, theoretical studies have been carried out with two-dimensional models, recently the study of blob dynamics has been approached by using three-dimensional numerical simulations, ${ }^{9,10}$ mainly focusing on the stability of blobs with respect to threedimensional instabilities, like drift-waves.

The goal of the present paper is to use three-dimensional simulations to study the dependence of the blob velocity on the plasma parameters, in particular, by exploring the different regimes of charge damping. We compare the blob velocity with the scaling based on the two-dimensional model and we explain the discrepancy through a detailed analysis of the three-dimensional simulation results. It is found that the two-dimensional scaling is well followed by the threedimensional simulations in the regime where charge damping is provided by the polarization current, while blobs move at a velocity that is higher than the two-dimensional prediction in the regime of parallel currents damping. This is due to the density drop observed in proximity of the sheath entrance, and the related decrease of the damping rate of the charge accumulation that is due to parallel currents.

The three-dimensional simulations are carried out with the GBS code. ${ }^{11}$ GBS solves the drift-reduced Braginskii equations (see, e.g., Ref. 11), with proper boundary conditions derived from first-principle analysis of the plasma sheath dynamics. ${ }^{12}$ GBS has been developed to simulate plasma dynamics in basic plasma physics devices, like linear machines and SMTs, ${ }^{13-16}$ and has been recently ported to the 
scrape-off layer (SOL) tokamak geometry. ${ }^{17-19}$ GBS is used here to simulate blob dynamics in the SMT geometry. In this toroidal magnetic configuration, characterized by helical field lines terminating on the vessel walls and resulting from the superposition of a small vertical magnetic field on the toroidal component, the main elements of blob dynamics are present in a simplified setting with respect to the tokamak SOL. In fact, the SMT features open field lines with oblique incidence at the vessel walls, with curvature and gradient of the magnetic field that are constant along a magnetic field line. Because of its relatively simplicity, the SMT constitutes an ideal test-bed where accurate measurements of blob velocity have been carried out spanning a large parameter range. ${ }^{7,8} \mathrm{We}$ consider seeded blob simulations, i.e., we follow the dynamics of a single blob that constitutes the initial condition for the simulations.

This paper is organized as follows. After introducing our model in Sec. II, in Sec. III, we briefly review blob dynamics, developing an analytical expression describing blob speed as a function of blob size. Sec. IV describes the threedimensional seeded blob simulations, focusing on understanding the mechanics of blob propagation and its relation to the analytical theory. The findings of our study are summarized in Sec. V.

\section{DRIFT-REDUCED BRAGINSKII EQUATIONS}

The plasma blob dynamics is studied using the driftreduced Braginskii model (see, e.g., Ref. 11). The model is able to describe plasma dynamics in open field configurations, where the high collision rate due to the low temperature tends to establish a local thermodynamic equilibrium. The driftreduced Braginskii equations can be deduced from the Braginskii fluid equations ${ }^{20}$ adopting the orderings $d / d t \ll \omega_{c i}$ ( $\omega_{c i}=e B / m_{i}$ is the ion gyrofrequency) and $k_{\perp} \gg k_{\|}$.

In the electrostatic, $T_{i} \ll T_{e}$ limit, the drift-reduced Braginskii equations derived in Ref. 11 are the continuity equation for the plasma density $n$, the equation for the vorticity $\omega$, the Ohm's law that determines the parallel electron velocity $v_{\| e}$, the equation for the parallel ion velocity $v_{\| i}$, and the equation for the electron temperature, $T_{e}$

$$
\frac{\partial n}{\partial t}=-R[\phi, n]-\nabla_{\|}\left(n v_{\| e}\right)+2\left[\hat{C}\left(p_{e}\right)-n \hat{C}(\phi)\right]+D_{n} \nabla_{\perp}^{2} n,
$$

$$
\begin{aligned}
\frac{\partial \omega}{\partial t}= & -R[\phi, \omega]-v_{\| i} \nabla_{\|} \omega+\frac{2}{n} \hat{C}\left(p_{e}\right)+\frac{1}{n} \nabla_{\|} j_{\|} \\
& +\frac{1}{3 n} \hat{C}\left(G_{i}\right)+D_{\omega} \nabla_{\perp}^{2} \omega,
\end{aligned}
$$

$$
\begin{aligned}
\frac{\partial v_{\| e}}{\partial t}= & -R\left[\phi, v_{\| e}\right]-v_{\| e} \nabla_{\|} v_{\| e}+D_{v_{\| e}} \nabla_{\perp}^{2} v_{\| e} \\
& +\frac{m_{i}}{m_{e}}\left\{\nu \frac{j_{\|}}{n}+\nabla_{\|} \phi-\frac{1}{n} \nabla_{\|} p_{e}-0.71 n \nabla_{\|} T_{e}-\frac{2}{3 n} \nabla_{\|} G_{e}\right\},
\end{aligned}
$$

$$
\begin{aligned}
\frac{\partial v_{\| i}}{\partial t}= & -R\left[\phi, v_{\| i}\right]-v_{\| i} \nabla_{\|} v_{\| i}-\frac{1}{n} \nabla_{\|} p_{e}-\frac{2}{3 n} \nabla_{\|} G_{i} \\
& +D_{v_{\| i}} \nabla_{\perp}^{2} v_{\| i},
\end{aligned}
$$

$$
\begin{aligned}
\frac{\partial T_{e}}{\partial t}= & -R\left[\phi, T_{e}\right]-v_{\| e} \nabla_{\|} T_{e}+\frac{4}{3} T_{e}\left[\frac{7}{2} \hat{C}\left(T_{e}\right)+\frac{T_{e}}{n} \hat{C}(n)-\hat{C}(\phi)\right] \\
& +\frac{2}{3} T_{e}\left[0.71 \nabla_{\|} v_{\| i}-1.71 \nabla_{\|} v_{\| e}\right. \\
& \left.+0.71\left(\frac{v_{\| i}-v_{\| e}}{n}\right) \nabla_{\|} n\right]+D_{T_{e}} \nabla_{\perp}^{2} T_{e}
\end{aligned}
$$

where the Poisson equation $\omega=\nabla_{\perp}^{2} \phi$ relates the vorticity with the electrostatic potential, $j_{\|}=n\left(v_{\| i}-v_{\| e}\right)$ is the parallel current, $R$ is here the torus major radius normalized to $\rho_{s}$, and $\nu=e^{2} n R /\left(m_{i} \sigma_{\|} c_{s}\right)$ is the normalized Spitzer resistivity. The $G_{e}$ and $G_{i}$ terms represent the gyroviscous part of the pressure tensor (see Ref. 11 for their explicit expressions). Small perpendicular diffusion terms of the form $D_{f} \nabla_{\perp}^{2} f$ are added for numerical reasons. In addition, $[f, g]=\mathbf{b} \cdot[\nabla f \times \nabla g]$ is the Poisson bracket, $\hat{C}(f)=B / 2[\nabla \times(\mathbf{b} / B)] \cdot \nabla f$ is the curvature operator, and $\nabla_{\|}=\mathbf{b} \cdot \nabla$ is the gradient in the direction parallel to the magnetic field. In addition, the Boussinesq approximation $\nabla \cdot\left(n \nabla_{\perp} \phi\right) \approx n \nabla_{\perp}^{2} \phi$ has been used to simplify the divergence of the polarization current (see, e.g., Refs. 21-23 for a discussion of the Boussinesq approximation).

We note that in Eqs. (1)-(5), as well as in the remainder of the present paper, $n$ is normalized to a reference value $n_{0}$, $T_{e}$ to a reference value $T_{e 0}, \phi$ to $T_{e 0} / e, v_{\| e}$ and $v_{\| i}$ to $c_{s 0}=\sqrt{T_{e 0} / m_{i}}$, and $t$ to $R / c_{s 0}$. Perpendicular and parallel lengths are normalized, respectively, to $\rho_{s 0}=c_{s 0} / \Omega_{c i}$ and $R$.

In the present study, we consider the SMT geometry, in which a vertical magnetic field $B_{v}$, superimposed on a toroidal field $B_{\varphi}$, creates helicoidal field lines with both ends terminating on the torus vessel. The number of field line turns in the torus is therefore $N=L_{v} / \Delta$, being $\Delta=2 \pi R B_{v} / B_{\varphi}$ the return distance of a field line in the poloidal plane. We denote the perpendicular coordinates with $x$ and $y$, being $x\left(0 \leq x \leq L_{x}\right)$ the radial coordinate, and $y\left(0 \leq y \leq L_{y}\right)$ the coordinate perpendicular to both $x$ and the magnetic field. In the limit of $B_{v} \ll B_{\varphi}$, considered herein, $y$ tends to correspond to the vertical direction. We use $\varphi$ for the periodic toroidal direction, $0 \leq \varphi<2 \pi$. In the SMT geometry, the expression for the operators assumes a relatively simple form: $\nabla_{\perp}^{2}=\partial_{x}^{2}+\partial_{y}^{2},[f, g]=\partial_{x} g \partial_{y} f-$ $\partial_{x} f \partial_{y} g, \hat{C}=\partial_{y}$ and $\nabla_{\|}=\partial_{\varphi}+\Delta /(2 \pi R) \partial_{y}$.

In Ref. 12, a set of boundary conditions is derived to describe the interface with the magnetic sheath interface, where the ion drift approximation, $d / d t \ll \omega_{c i}$, breaks down. The boundary conditions, used here at the top and bottom ends of the vertical domain, are

$$
\begin{gathered}
v_{\| i}= \pm c_{s}, \\
v_{\| e}= \pm c_{s} \exp \left(\Lambda-\phi / T_{e}\right), \\
\frac{\partial n}{\partial y}=\mp \frac{n}{c_{s}} \frac{\partial v_{\| i}}{\partial y}, \\
\omega=-\cos ^{2}\left(\frac{\Delta}{2 \pi R}\right)\left[\left(\frac{\partial v_{\| i}}{\partial y}\right)^{2} \pm c_{s} \frac{\partial^{2} v_{\| i}}{\partial y^{2}}\right], \\
\frac{\partial \phi}{\partial y}=\mp c_{s} \frac{\partial v_{\| i}}{\partial y},
\end{gathered}
$$




$$
\frac{\partial T_{e}}{\partial y}=0
$$

where $\Lambda \approx 3$.

We note that the SMT magnetic configuration can also be visualized as a flux tube that wraps around the torus $N$ times, with vertical extensions $\Delta$ and length in the parallel direction $2 \pi N$. As a matter of fact, as blobs are structures aligned to the magnetic field, in order to describe more conveniently their dynamics, we also introduce a flux tube coordinate system, $(\chi, \xi, \zeta): \chi$ coincides with the radial coordinate $x ; \xi$ matches the vertical direction and extends over the vertical extension of the flux tube, $-\Delta / 2 \leq \xi<\Delta / 2$; and $\zeta$ is a coordinate parallel to the magnetic field, defined such that its origin, $\zeta=0$, is at the position for which $\varphi=0$ that is closest to the field line midpoint.

The drift-reduced equations were implemented in GBS, ${ }^{11}$ a turbulence code that was first developed to study plasma turbulence in basic plasma physics devices, in particular, in linear devices such as LAPD ${ }^{24}$ and in the SMT geometry such as TORPEX, ${ }^{13-16}$ and it has now been ported to the tokamak SOL geometry. ${ }^{17-19}$ A validation exercise of GBS results has been performed against experimental data from the TORPEX device. ${ }^{16,25}$ In GBS, spatial derivatives are expressed using second-order finite difference scheme, except for the Poisson bracket terms that are described with the Arakawa scheme. ${ }^{26}$ Time advance is performed with a standard fourth order Runge-Kutta solver. The detailed description of the algorithm used by GBS is presented in Ref. 11.

\section{BLOB VELOCITY SCALING WITH SIZE}

It is useful, in order to aid the interpretation of the threedimensional blob simulations, to briefly review the physical effects governing blob propagation. We develop a blob velocity scaling as a function of the blob size, which can then be compared against our simulation results. We first note that, by neglecting gyroviscous effects, the vorticity equation in the drift-reduced model, Eq. (2), can be written as $\left(\nabla \cdot \mathbf{j}_{\star}+\nabla \cdot \mathbf{j}_{\|}+\nabla \cdot \mathbf{j}_{\text {pol }}\right) / n=0$, i.e., it involves the sum of the divergence of the diamagnetic, parallel, and ion polarization currents. This form of the vorticity equation highlights the principal driving and damping mechanisms for blob motion. The diamagnetic flow is almost incompressible, and only a term associated to magnetic field curvature enters the dynamics, the so-called interchange drive, $\nabla \cdot \mathbf{j}_{\star}=2 \hat{C}\left(p_{e}\right)$. This term drives a dipole structure and constitutes the driving mechanism for blob propagation. The parallel current flows along the magnetic field lines, weakening the curvaturedriven dipole and damping the blob motion. The remaining charge must then be compensated by the flow of the polarization current, which eventually gives rise to secondary instability. The polarization current contribution is expressed as $\nabla \cdot \mathbf{j}_{\text {pol }} / n=\partial_{t} \omega+R[\phi, \omega]-v_{\| i} \nabla_{\|} \omega$.

In order to evaluate the contribution of the parallel current term, $\nabla \cdot \mathbf{j}_{\|}$, we assume a weak dependence of the plasma properties in the direction parallel to the magnetic field lines, and we integrate the vorticity equation in this direction. The parallel current contribution to the vorticity is integrated along the field line, reducing to the contribution of the sheath currents, $j_{\|}^{ \pm}= \pm \sigma n c_{s}\left[1-\exp \left(\Lambda-\phi / T_{e}\right)\right]$. Here, $n c_{s}$ refers to the density and sound speed at the center of the blob and $\sigma$ denotes the $n c_{s}$ drop from the center of the blob to the sheath entrance (it is typically assumed $\sigma \simeq 1 / 2$ ). This yields $j_{\|} \approx 2 \sigma n c_{s} \tilde{\phi}$, having assumed relatively small perturbation from the equilibrium state, i.e., $\left[1-\exp \left(\Lambda-\phi / T_{e}\right)\right] \sim \phi-\Lambda T_{e}=\tilde{\phi}$, where $\tilde{\phi}$ is the floating potential. The resulting model is as follows:

$$
\frac{\partial \omega}{\partial t}+R[\phi, \omega]=\frac{2}{p} \frac{\partial p}{\partial y}-2 \frac{\sigma n c_{s}}{L_{c}} \tilde{\phi} .
$$

The density and temperature equations are combined, obtaining an equation for the pressure, which can help estimating the magnitude of the diamagnetic currents. The leading order terms yield

$$
\frac{\partial p}{\partial t}+R[\phi, p]=0
$$

The derivation of the scaling of the blob velocity now closely follows Ref. 7. We assume that the blob is a pressure monopole associated to a potential dipole. Since $\mathbf{E} \times \mathbf{B}$ drifts propel the blob motion, it can be inferred that $\tilde{\phi} \sim \phi$ $\sim v_{\perp} a_{\perp}$. The terms of Eq. (12) are now approximated at the maximum positive potential of the dipole using the following expressions: $\partial p / \partial y \sim-p_{b} / a_{\perp}, \nabla_{\perp} \phi=0, \omega \sim-\phi / a_{\perp}^{2}$, and $\partial_{t}+R[\phi,] \sim \gamma_{\text {int }}$ ( $p_{b}$ is the blob peak pressure in excess of the background pressure $p_{0}$ and $\gamma_{i n t}=\sqrt{2 R / a_{\perp}}$ is the reference interchange growth rate). Defining $v_{0}=\left(2 L_{c} / R^{2}\right)^{1 / 5}$ and using $a_{0}=\left(4 L_{c}^{2} R\right)^{1 / 5}$, we recover the expression

$$
v_{\perp}=v_{0}\left[\frac{\sqrt{2 a_{\perp} / a_{0}}}{1+2 \sqrt{2} \sigma\left(a_{\perp} / a_{0}\right)^{5 / 2}}\right] \frac{p_{b}}{p_{0}+p_{b}} .
$$

It is readily verifiable, from Eq. (14), that $v_{\perp} \sim \sqrt{a_{\perp}}$ in the limit $a_{\perp} \ll 1$ and, conversely, that $v_{\perp} \sim 1 / a_{\perp}^{2}$ in the limit $a_{\perp} \gg 1$. The size of the fastest moving blobs can be found by optimizing $v_{\perp}$ with respect to $a_{\perp} / a_{0}$, which gives $a_{\perp} / a_{0}=\left(18 \sigma^{2}\right)^{-1 / 5} \approx 1$.

\section{THREE-DIMENSIONAL SIMULATIONS OF SEEDED BLOBS}

We carry out simulations of a seeded blob structure localized in the perpendicular plane and elongated in the parallel direction. The initial condition for the density and temperature profiles is given by

$$
n=n_{0}+n_{b} f_{b}
$$

and

$$
T=T_{0}+T_{b} f_{b},
$$

where

$$
f_{b}(\chi, \xi, \zeta)=\exp \left\{-\log 2\left[\frac{\left(\chi-\chi_{0}\right)^{2}+\xi^{2}}{a_{\perp}^{2}}+\frac{\zeta^{2}}{a_{\|}^{2}}\right]\right\}
$$


and $n_{0}, n_{b}, T_{0}$, and $T_{b}$ are the background and blob densities and temperatures, respectively. The blob is initially centered at the radial position $\chi=\chi_{0}$, and $a_{\perp}$ and $a_{\|}$define the initial perpendicular and parallel blob sizes. The initial condition is defined in such a way that the blob density drops to $n=n_{0}+n_{b} / 2$ at the contour where $\left[\left(\chi-\chi_{0}\right)^{2}+\xi^{2}\right] / a_{\perp}^{2}+\zeta^{2} / a_{\|}^{2}=1$. Therefore, the blob sizes $a_{\perp}$ and $a_{\|}$indicate the half-width of the half-maximum density or temperature of the blob in the perpendicular and parallel directions.

The electric potential is initialized such that a vertical dipole structure is created around the blob. A small, radial, outward $\mathbf{E} \times \mathbf{B}$ velocity localized at the center of the blob results upon initialization. The initial potential is given by the expression

$$
\phi=-\frac{\partial n}{\partial y} .
$$

We have carried out a sensitivity study to evaluate the choice of initial condition for $\phi$, finding no discernible effect in the dynamics after $t=0.01 R / c_{s}$ due to the strength of the initial dipole. Since in TORPEX geometry, we expect that blobs originate from an interchange instability, which is characterized by a dipole potential structure, we did not attempt initial
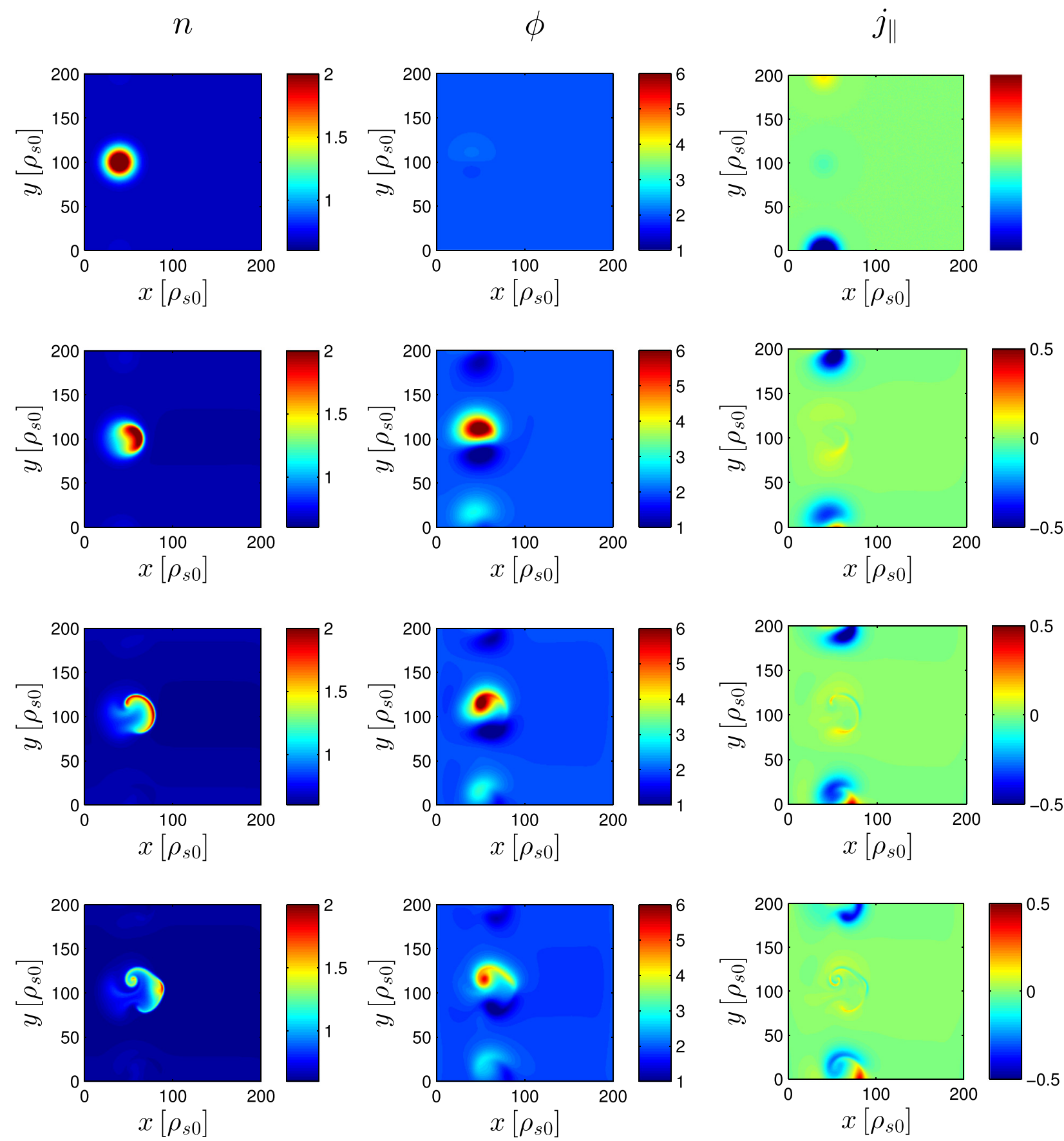

FIG. 1. Perpendicular plane snapshots of $n$ (left), $\phi$ (center), and $j_{\|}$(right) are plotted (top to bottom) at $t=0,0.24,0.48$, and $0.72 R / c_{s}$ at the poloidal plane defined by $\zeta=0$. The blob is initialized with $a_{\perp}=a_{0}, a_{\|}=L_{c} / 4$. 
conditions such as $\phi=0$ or $\phi \propto n$. The initial conditions for the parallel velocities are obtained by requiring a balance between the leading order terms in Ohm's law, Eq. (3), that also satisfies Bohm's condition at the boundaries. The parallel velocities are initialized as follows:

$$
\begin{gathered}
v_{\| i}=c_{s}\left(\frac{2 y}{L_{v}}-1\right), \\
v_{\| e}=v_{\| i}-\frac{1}{\nu n}\left\{\nabla_{\|} \phi-\frac{T_{e}}{n} \nabla n-1.71 \nabla_{\|} T_{e}\right\},
\end{gathered}
$$

where $c_{s}$ is here the sound speed at the magnetic presheath entrance, as given by the initial temperature profile, Eq. (16). Equations (15)-(20) together with the Poisson equation $\omega=$ $\nabla_{\perp}^{2} \phi$ define all the initial conditions required to carry out three-dimensional simulations of blob dynamics.

The following parameters are used herein to simulate SMT blob dynamics: $R=500, L_{v}=L_{x}=200, N=2, \nu=0.1$, which give approximately $n_{0} \approx 10^{17} \mathrm{~m}^{-3}$ and $T_{0} \approx 1 \mathrm{eV}$. The grid resolution is $n_{z}=64$ in the toroidal direction and $n_{v}=n_{x}=256$ in the perpendicular plane, which results in 32 toroidal modes and a vertical and horizontal resolution of $0.78 \rho_{s 0}$. The time step used for Runge-Kutta integration is $\Delta t=10^{-4}$. The initial blob position is $\chi_{0}=40$, $n_{0}=T_{0}=0.67, n_{b}=T_{b}=2$, and we consider the following perpendicular sizes: $a_{\perp}=\{0.2,0.4,0.6,0.8,1,1.2,1.4,1.6$, $1.8,2\} a_{0}$, where $a_{0}=\left(4 L_{c}^{2} R\right)^{1 / 5} \approx 13$ is the reference blob size, the connection length for this SMT configuration being given by $L_{c}=2 \pi N=4 \pi$. For each $a_{\perp}$, three different longitudinal sizes have been considered, $a_{\|}=\{1 / 4,1,5\} L_{c}$, respectively, corresponding to detached, connected, and flute-like initial conditions.

As an example, we discuss the propagation of a blob with $a_{\perp}=a_{0}$ and $a_{\|}=L_{c} / 4$, which is typically the most structurally stable size. Snapshots of $n$ (left), $\phi$ (center), and $j_{\|}$(right) are shown in Fig. 1. Starting from its initial position, the blob propagates in the direction of the outer wall of the SMT, propelled by an $\mathbf{E} \times \mathbf{B}$ drift resulting from a potential dipole structure. As the blob accelerates, it rapidly deforms into a mushroom-shaped structure due to the vertical variation of the dipole strength, which results in a higher velocity at the center of the structure. Following this phase of the dynamics, turbulent structures form, leading to the $n$
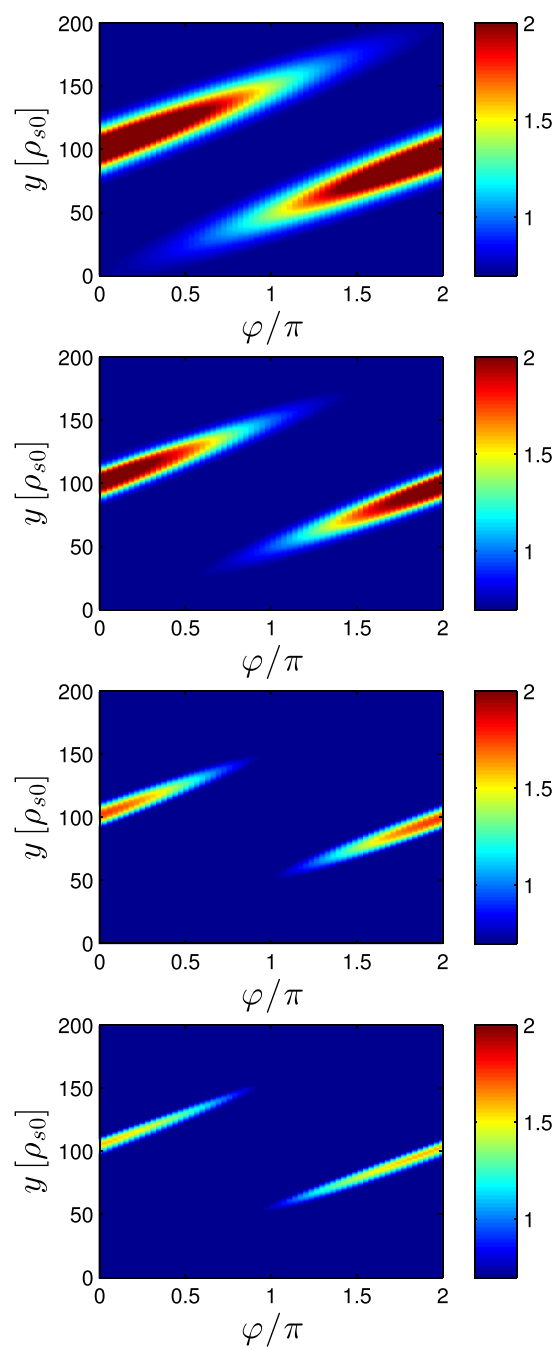

$\phi$
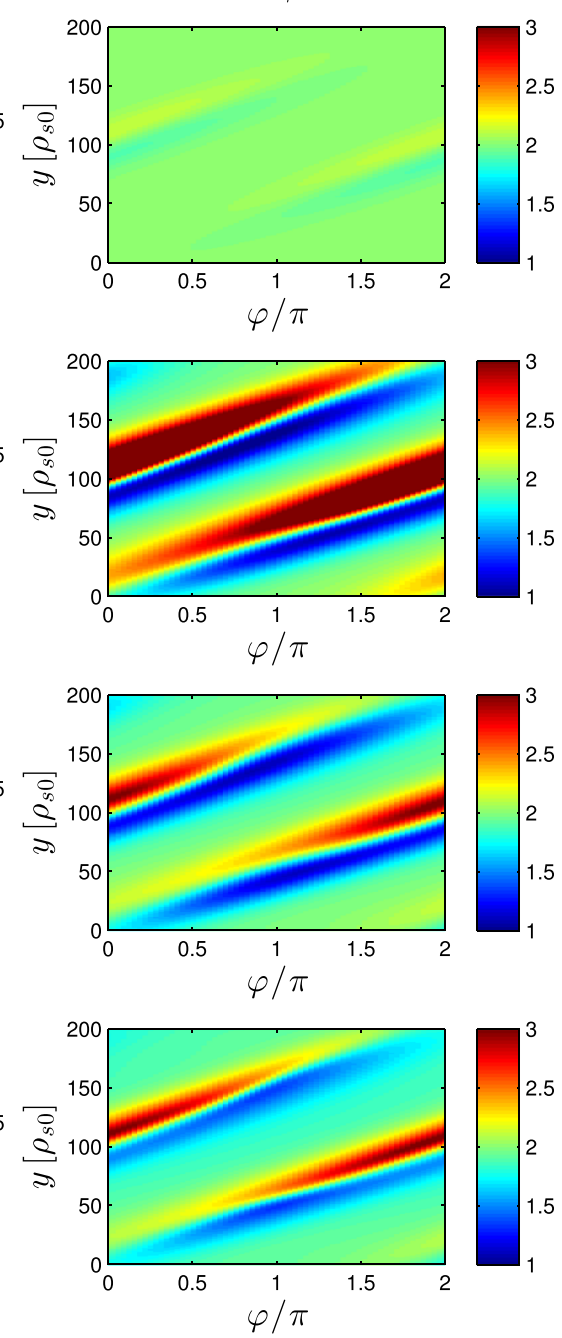
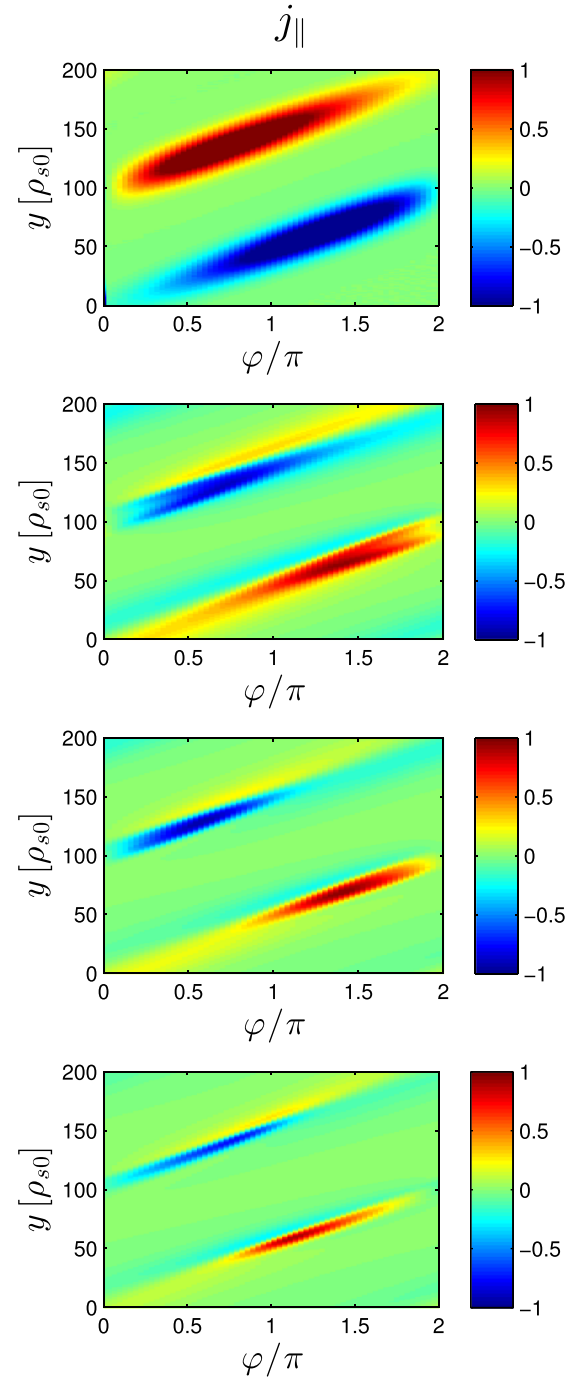

FIG. 2. Parallel plane snapshots of $n$ (left), $\phi$ (center), and $j_{\|}$(right) are plotted (top to bottom) at $t=0,0.24,0.48$, and $0.72 R / c_{s}$. The blob is initialized with $a_{\perp}=a_{0}, a_{\|}=L_{c} / 4$. 

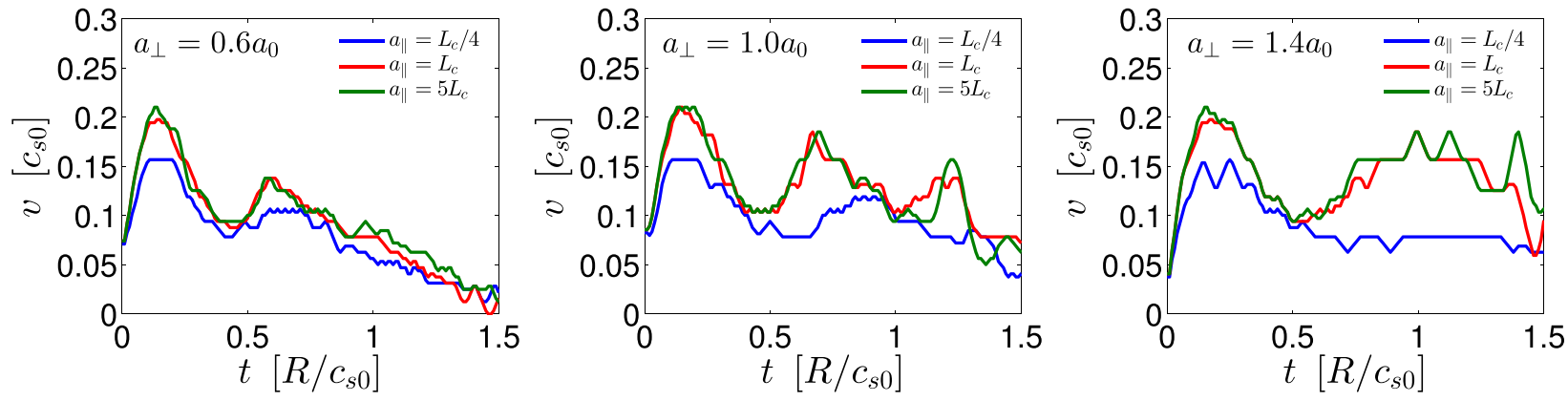

FIG. 3. Instantaneous blob front velocity as a function of time is shown for simulations with initial blob sizes $a_{\perp}=0.6,1.0$, and $1.4 a_{0}$ (left, center, and right panels, respectively). In each case, the velocity is plotted for $a_{\|}=L_{c} / 4, L_{c}, 5 L_{c}$ (blue, red, and green lines, respectively).

break-up of the blob. During this time, the blob can travel a significant portion of the radial extension of the vessel.

There is an upward motion of the blob structures that is more noticeable in cases with $a_{\|}=L_{c} / 4$. We also note that all turbulent structures appear to remain approximately field aligned, i.e., the presence of drift wave instability is not easily identifiable. In contrast with Refs. 9 and 10, here the blob propagation and stability appear to be only moderately affected by $3 \mathrm{D}$ effects such as drift waves or the Boltzmann rotation effect.

The typical dipole structure of the potential remains during the blob propagation, although an asymmetry in $\phi$ between the upper and lower lobe forms. The parallel current associated with the blob, plotted on the right column of Fig. 1, becomes very small where the blob density peaks. In fact, most of the current seems to be driven near the sheath entrance.

In order to illustrate the parallel dynamics, we plot $n, \phi$, and $j_{\|}$in Fig. 2 on the plane parallel to the peak blob density. These snapshots show the blob winding around the torus with periodicity $N=2$. The potential dipole is strongest close to where the density peaks, about $L_{c} / 2$ away from the sheath. The parallel current appears to drive a closed circuit exchanging charge between the sheaths and the blob, with $j_{\|}$ being negligible near the blob density peak.

We focus our analysis on the blob radial velocity, which is calculated as the time rate of change of the blob front position. The latter is defined as the horizontal point of maximum $\partial_{\chi} n$ within the vertical range $-2 a_{\perp}<\xi<2 a_{\perp}$. For simplicity, we have considered the motion in the poloidal plane defined by $\varphi=0$. Figure 3 shows the instantaneous blob speed as a function of time for simulations with $a_{\perp}=\{0.6$, $1,1.4\} a_{0}$ (left, center, right), which allows us to make some observations concerning blob propagation. There is an initial maximum in the speed, which is mainly due to the displacement of the blob peak towards the front. Occasionally, there are additional peaks which can result from mass ejections or even a splitting of the blob. The blob with $a_{\perp}=0.6$ propagates for roughly $0.7 R / c_{s}$ before it is destroyed by secondary instability, while the larger blobs are able to propagate for longer times.

Figure 4 shows the mean blob velocity, $v_{b}$, as a function of the initial sizes $a_{\perp}$ and $a_{\|}$. Obtaining $v_{b}$ involves a time average that avoids the initial acceleration stage and the final stage when the blobs become unstable and break up. As expected from Eq. (14), $v_{b}$ peaks around $a_{\perp}=a_{0}$. The propagation of the blob is damped at small sizes by the polarization current and at larger sizes by the parallel current.

The predictions of Eq. (14) are superimposed in Fig. 4. Note that, since the simulations are carried out using a global three-dimensional model, the sheath currents evolve selfconsistently with the plasma dynamics, leading to $\sigma<1 / 2$. For blobs with $a_{\|} \gtrsim L_{c}$, we observe $\sigma \approx 1 / 8$ and $p_{b} /\left(p_{b}+\right.$ $\left.p_{0}\right) \approx 0.93$, while blobs with $a_{\|}=L_{c} / 4$ we observe $\sigma \approx 1 / 6$ and $p_{b} /\left(p_{b}+p_{0}\right) \approx 0.89$. The analytical scaling, Eq. (14), matches the simulation data very well, especially for $a_{\|} \gtrsim L_{c}$. In addition, we have plotted the analytical scaling obtained using the commonly used approximation $\sigma=1 / 2$, finding that $v_{\perp}$ is underestimated by a factor of 4 for large $a_{\perp} / a_{0}$. In fact, the parallel current damping term in our simulations is much weaker than expected from the previous twodimensional simulations, ${ }^{8}$ where an analytical form for the sheath current with $\sigma=1 / 2$ is imposed in the vorticity equation.

In order to gain further insight into our simulation results, we have reconstructed Eq. (2) by applying finite difference formulas on the simulation data to reconstruct $\nabla \cdot \mathbf{j}_{\star} / n$ and $\nabla \cdot \mathbf{j}_{\|} / n$. The time derivative of the vorticity, which represents the divergence of the polarization current, is obtained from $\nabla \cdot \mathbf{j}_{\text {pol }} / n=-\nabla \cdot\left(\mathbf{j}_{\star}+\mathbf{j}_{\|}\right) / n$. (Additionally,

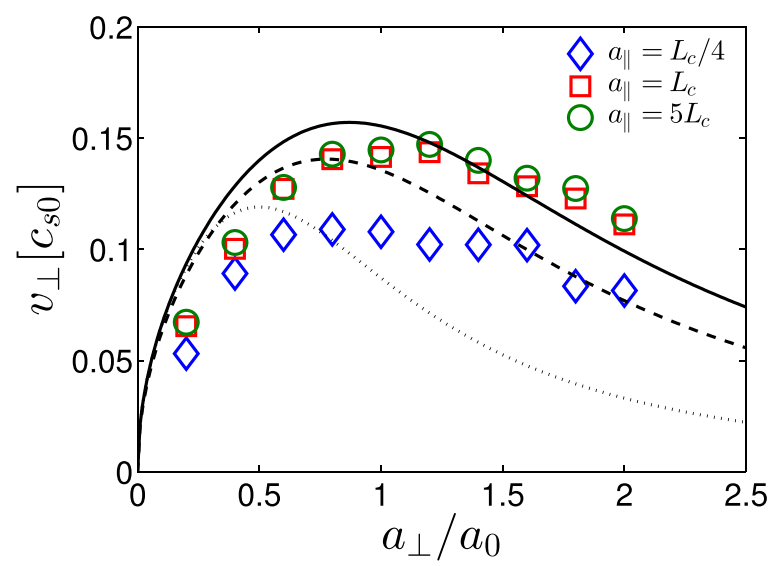

FIG. 4. Mean velocities obtained from GBS seeded blob simulations with $a_{\perp}=0.2-2.0 a_{0}, a_{\|}=L_{c} / 4, L_{c}, 5 L_{c}$ (blue diamonds, red squares, and green circles, respectively) are compared against the predictions stemming from Eq. (14) using: $\sigma=1 / 8$ with $p_{b} /\left(p_{b}+p_{0}\right)=0.93$ (solid line); $\sigma=1 / 6$ with $p_{b} /\left(p_{b}+p_{0}\right)=0.89$ (dashed line); and finally, $\sigma=1 / 2$ with $p_{b} /\left(p_{b}+p_{0}\right)=0.93($ dotted line $)$. 

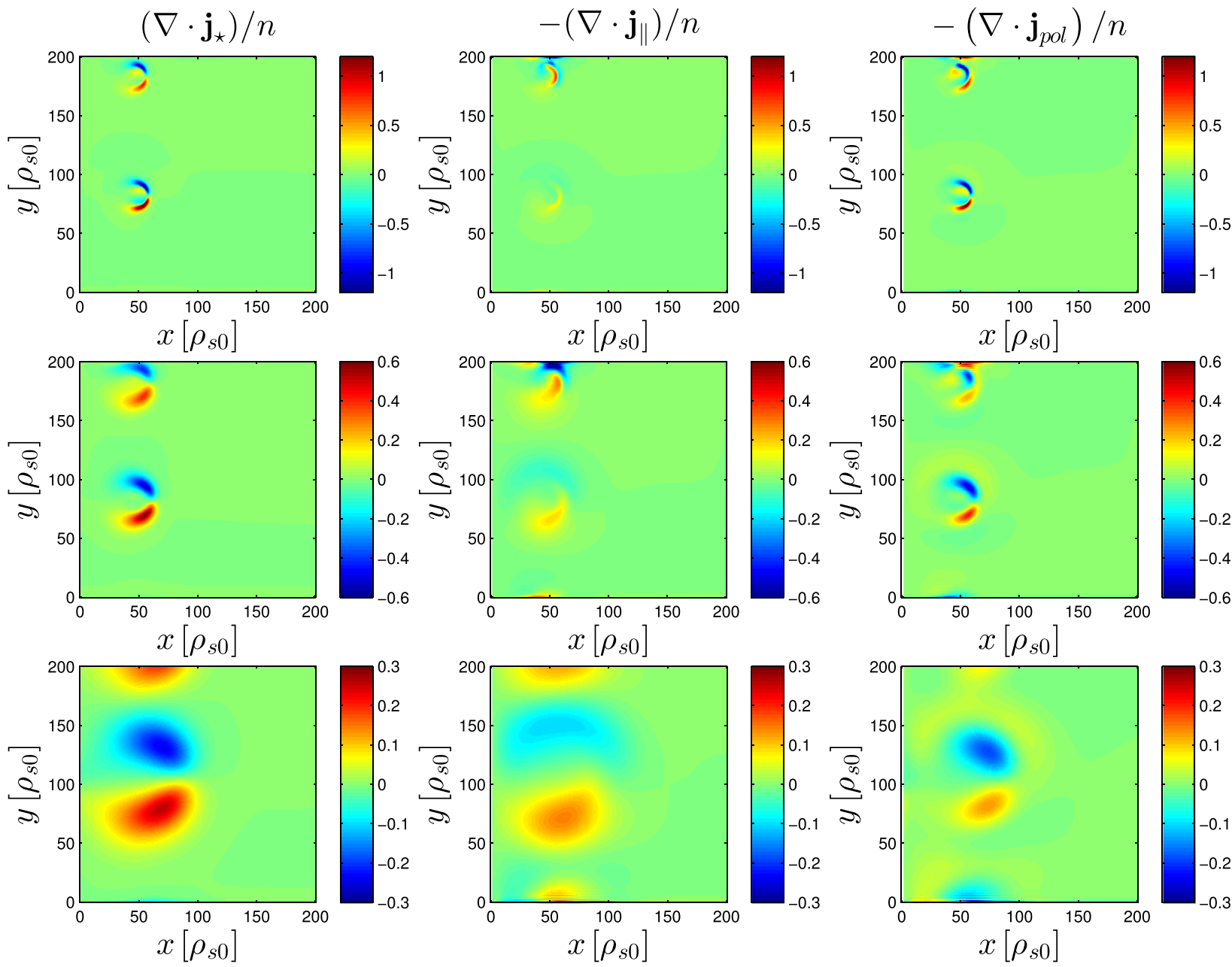

FIG. 5. Balance between the divergence of diamagnetic, parallel, and polarization currents (left, center, and right columns) is plotted for simulations with $a_{\perp}=0.6 a_{0}, 1.0 a_{0}, 1.8 a_{0}$ (top, center, and bottom rows) and $a_{\|}=L_{c}$ at $t=0.16, \varphi=5 / 3 \pi$.

we have verified that the diffusion term $D_{\omega} \nabla_{\perp}^{2} \omega$ is negligible with respect to the divergence of the considered currents.) This procedure allows us to evaluate the different components of the vorticity equation.

As an example, in Fig. 5, we plot separately the curvature, parallel damping, and polarization terms (left, center, and right columns) for blobs with $a_{\perp}=0.6 a_{0}, 1.0 a_{0}, 1.8 a_{0}$ (top, center, and bottom rows) and $a_{\|}=L_{c}$ at $t=0.16$. We choose the perpendicular plane defined by $\varphi=5 \pi / 3$ in order to show one portion of the blob near its peak alongside another part of the blob near the sheath. The curvature drive (left column) is balanced by the sum of parallel damping and
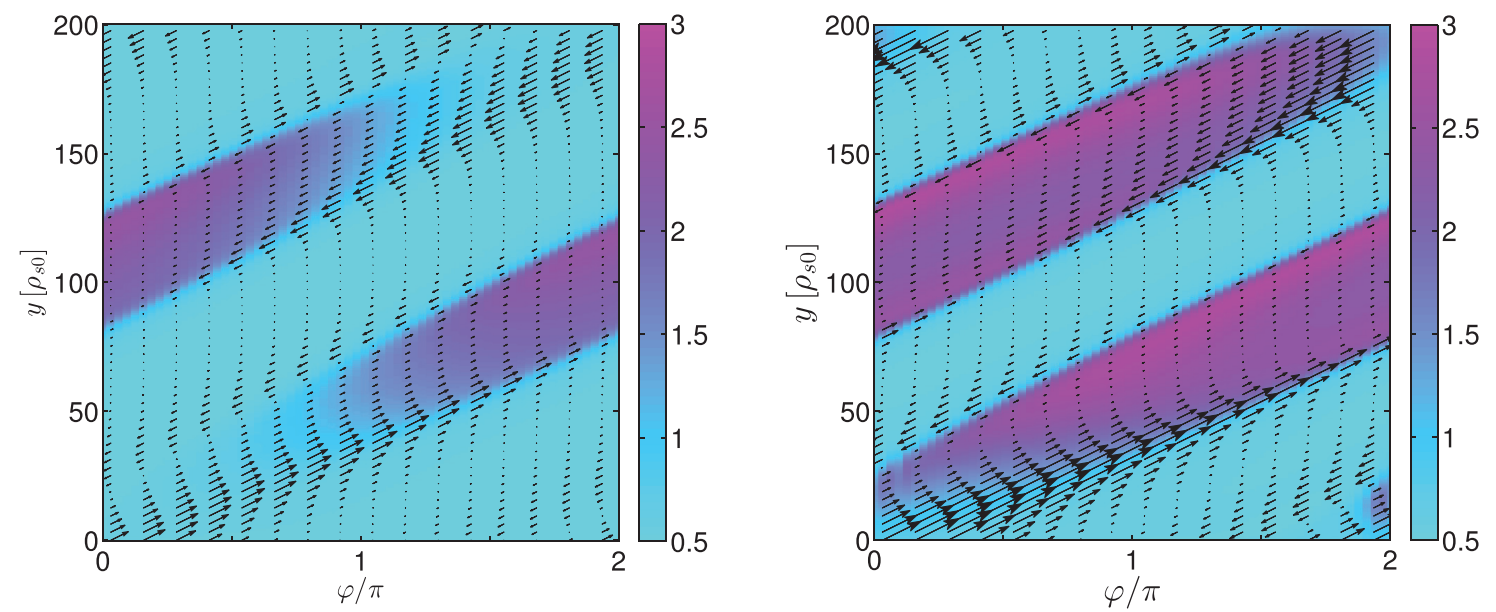

FIG. 6. Parallel current (toroidal plane view at $x=82$ ) is plotted as a vector field at $t=0.48$ for blobs of size $a_{b}=1.8, a_{\|}=L_{c} / 4$ (left) and $a_{\|}=L_{c}$ (right). The color indicates the magnitude of $n c_{s}$. 
polarization terms (center and right rows). First, we note an asymmetry of the damping mechanisms between the upper and lower lobes near the blob peak. In the upper lobe, the curvature term is balanced almost completely by the polarization current, while on the lower lobe there is a similar contribution from both damping mechanisms. Second, we observe that as the blob size increases, the parallel current damping term becomes larger. It is estimated that about $60 \%$ of the curvature drive is balanced by parallel currents, although the polarization effect remains significant. Notably, it is observed that polarization currents remain important even at large blob sizes, a symptom of the decreased importance of the parallel current damping observed in our simulations.

Finally, the flow of parallel currents along the blob structure is plotted in Fig. 6 at $x=82$. The background color represents $n c_{s}$, while the arrows indicate the magnitude and direction of the current. The parallel current attains a large magnitude near the sheath and a significant drop of $n c_{s}$ is observed. The density losses are stronger at the lower sheath, with $\sigma \approx 1 / 8$. Using the plotted quantities, we have evaluated $\nabla_{\|} j$ as well as the linearized expression for the sheath current term $\nabla_{\| j_{\|}} \sim n c_{s} \phi-\Lambda T_{e}$. Both of these results are consistent with the use of $\sigma \approx 1 / 8$ in Eq. (14).

\section{CONCLUSIONS}

The propagation of single-seeded blobs in the SMT geometry has been studied using global three-dimensional non-linear simulations (Eqs. (1)-(5)). We have carried out a simulation scan in order to understand the scaling of the blob velocity respect to blob size.

By considering the blob sizes $a_{\perp}=0.2-2.0 a_{0}$ and $a_{\|}=L_{c} / 4, L_{c}, 5 L_{c}$, we find that the blob propagation mechanisms in three-dimensional simulations are akin to their two-dimensional counterparts: the interchange drive creates and sustains an electric dipole that propels the blob forward. The dipole is then partially compensated by parallel and polarization currents, which regulate the maximum attainable speed. As we would expect from the analytical theory, blobs are fastest when $a_{\perp} \approx a_{0}$, and in this case $v_{\perp} \sim v_{0}$.

In particular, a generalization of the $v_{\perp}$ scaling given in Ref. 7, derived for arbitrary $n c_{s}$ losses at the sheath, is compared to the simulation results. In order to derive this scaling, the vorticity equation is integrated along the magnetic field lines, paying special attention to the parallel current damping term. In principle, it is sufficient to use the typical sheath current estimate $\nabla_{\|} j_{\|} \approx 2 \sigma n c_{s} \phi / L_{c}$, provided that a good estimate of $\sigma$ is available. The dimensionless scaling arising from this model, Eq. (14), matches the simulation data very well, provided that the value of $\sigma$ found in the non-linear simulations is used. We have found, however, that the common assumption $\sigma=1 / 2$ is in fact not fulfilled in our simulations. This approximation gives rise to a large overestimate of the parallel current damping at large blob sizes.

A detailed balance of the divergence of the currents has been shown. As expected, polarization currents play an important role when $a_{\perp}<a_{0}$, while parallel currents become more important when $a_{\perp} \gtrsim 1$. However, we have found that the effect of parallel currents is weaker than in the twodimensional case, where the sheath current is imposed as a damping term in the vorticity equation. In our simulations, on the other hand, the density and the parallel current evolve self-consistently. The result is that a current circuit forms between the sheaths and the blob, carrying much of the plasma towards the plasma facing components and causing parallel density losses far surpassing the $\sigma=1 / 2$ assumption. As a consequence, the interchange drive is reduced near the sheath, and parallel currents in this region are partially balanced by polarization currents. The polarization current drives secondary instability, reinforcing the density losses. In fact, the blob is almost immediately destroyed near the sheath, and the initial $a_{\|}$plays essentially no role in the dynamics provided that $a_{\|} \gtrsim L_{c}$. Altogether, these effects result in a considerably faster blob motion in three-dimensional simulations, when compared with previous two-dimensional work.

\section{ACKNOWLEDGMENTS}

We acknowledge useful discussions with A. Fasoli, I. Furno, and C. Theiler. Part of the simulations presented herein was carried out at the Swiss National Supercomputing Centre (CSCS) under Project ID s346; and part was carried out using the HELIOS supercomputer system at Computational Simulation Centre of International Fusion Energy Research Centre (IFERC-CSC), Aomori, Japan, under the Broader Approach collaboration between Euratom and Japan, implemented by Fusion for Energy and JAEA. This research was supported by the Swiss National Science Foundation.

${ }^{1}$ S. Krasheninnikov, Phys. Lett. A 283, 368 (2001).

${ }^{2}$ D. A. D’Ippolito, J. R. Myra, and S. I. Krasheninnikov, Phys. Plasmas 9 , 222 (2002).

${ }^{3}$ D. A. D'Ippolito, J. R. Myra, and S. J. Zweben, Phys. Plasmas 18, 060501 (2011).

${ }^{4}$ S. I. Krasheninnikov, D. A. D’Ippolito, and J. R. Myra, J. Plasma Phys. 74, 679 (2008)

${ }^{5}$ A. Fasoli, B. Labit, M. McGrath, S. H. Muller, G. Plyushchev, M. Podesta, and F. M. Poli, Phys. Plasmas 13, 055902 (2006).

${ }^{6}$ A. Fasoli, A. Burckel, L. Federspiel, I. Furno, K. Gustafson, D. Iraji, B. Labit, J. Loizu, G. Plyushchev, P. Ricci, C. Theiler, A. Diallo, S. H. Mueller, M. Podestá, and F. Poli, Plasma Phys. Controlled Fusion 52, 124020 (2010).

${ }^{7}$ C. Theiler, I. Furno, P. Ricci, A. Fasoli, B. Labit, S. H. Müller, and G. Plyushchev, Phys. Rev. Lett. 103, 065001 (2009).

${ }^{8}$ I. Furno, C. Theiler, D. Lançon, A. Fasoli, D. Iraji, P. Ricci, M. Spolaore, and N. Vianello, Plasma Phys. Controlled Fusion 53, 124016 (2011).

${ }^{9}$ J. R. Angus, M. V. Umansky, and S. I. Krasheninnikov, Phys. Rev. Lett. 108, 215002 (2012).

${ }^{10}$ N. R. Walkden, B. D. Dudson, and G. Fishpool, Plasma Phys. Controlled Fusion 55, 105005 (2013).

${ }^{11}$ P. Ricci, F. D. Halpern, S. Jolliet, J. Loizu, A. Mosetto, A. Fasoli, I. Furno, and C. Theiler, Plasma Phys. Controlled Fusion 54, 124047 (2012).

${ }^{12}$ J. Loizu, P. Ricci, F. D. Halpern, and S. Jolliet, Phys. Plasmas 19, 122307 (2012).

${ }^{13}$ P. Ricci, B. N. Rogers, and S. Brunner, Phys. Rev. Lett. 100, 225002 (2008).

${ }^{14}$ P. Ricci and B. N. Rogers, Phys. Plasmas 16, 062303 (2009).

${ }^{15}$ P. Ricci and B. N. Rogers, Phys. Rev. Lett. 104, 145001 (2010).

${ }^{16}$ P. Ricci, C. Theiler, A. Fasoli, I. Furno, K. Gustafson, D. Iraji, and J. Loizu, Phys. Plasmas 18, 032109 (2011). 
${ }^{17}$ F. D. Halpern, S. Jolliet, J. Loizu, A. Mosetto, and P. Ricci, Phys. Plasmas 20, 052306 (2013).

${ }^{18}$ A. Mosetto, F. D. Halpern, S. Jolliet, J. Loizu, and P. Ricci, Phys. Plasmas 20, 092308 (2013).

${ }^{19}$ F. Halpern, P. Ricci, B. Labit, I. Furno, S. Jolliet, J. Loizu, A. Mosetto, G. Arnoux, J. Gunn, J. Horacek, M. Kočan, B. LaBombard, C. Silva, and JET-EFDA Contributors, Nucl. Fusion 53, 122001 (2013).

${ }^{20}$ S. I. Braginskii, Transport Processes in a Plasma, Reviews of Plasma Physics, Vol. 1, edited by M. A. Leontovich (Consultants Bureau, New York, 1965), p. 205.
${ }^{21}$ G. Q. Yu, S. I. Krasheninnikov, and P. N. Guzdar, Phys. Plasmas 13, 042508 (2006).

${ }^{22}$ K. Bodi, G. Ciraolo, P. Ghendrih, F. Schwander, E. Serre, and P. Tamain, in 38th EPS Conference on Plasma Physics, Strasbourg, France (2011), p. P1.121.

${ }^{23}$ D. Russell, D. D'Ippolito, and J. Myra, Bull. Am. Phys. Soc. 57, BP8.00159 (2012), see http://meetings.aps.org/link/BAPS.2012.DPP.BP8.159.

${ }^{24}$ B. N. Rogers and P. Ricci, Phys. Rev. Lett. 104, 225002 (2010).

${ }^{25}$ P. Ricci, C. Theiler, A. Fasoli, I. Furno, B. Labit, S. H. Muller, M. Podesta, and F. M. Poli, Phys. Plasmas 16, 055703 (2009).

${ }^{26}$ A. Arakawa, J. Comput. Phys. 1, 119 (1966). 